\title{
Effects of Dispersion Fiber on CWDM Directly Modulated System Performance
}

\author{
Carmina del Río Campos and Paloma R. Horche \\ Escuela Politécnica Superior, Universidad San Pablo CEU \\ ETSIT, Universidad Politécnica de Madrid \\ Spain
}

\section{Introduction}

The Coarse Wavelength Division Multiplexing technology (CWDM) enables carriers to transport more services over their existing optical fiber infrastructure by combining multiple wavelengths onto a single optical fiber. CWDM is technologically simpler and easier to implement and is a good fit for access networks and many metro/regional networks. ITU-T G.694.2 defines 18 wavelengths for CWDM, using the wavelengths from $1270 \mathrm{~nm}$ through $1610 \mathrm{~nm}$ with a channel spacing of $20 \mathrm{~nm}$. This channel spacing allows to use, in CWDM systems, low-cost and uncooled lasers, e.g. direct modulated laser (DML).

The high output power of commercial 1.55- $\mu \mathrm{m}$ DMLs can provide a power budget that allows for amplifier/regenerator spacing of $80-100 \mathrm{~km}$. However, the frequency chirp characteristics of DMLs significantly limit the maximum achievable transmission distance over standard single-mode fibers (SMF) [Cartledge 1989].

A number of approaches have been used to improve transmission performance using directly modulated lasers, including cutting down the chirp externally using a narrow bandpass filter [Yan 2005] and the deployment of a negative dispersion fiber [Tomkos 2001a, Tomkos 2002]. However, typical metro and access networks are made up of a conventional single-mode fiber (SMF) and because of the cost and difficulty (or lack of feasibility) in changing embedded fiber links, a method that enhances system performance requiring only the modification of one or both of the endpoints of a link is a critical requirement.

In this chapter, by means of a commercial Optical Communication System Design Software, we evaluate the interaction of fiber dispersion with the laser chirp in context of positive and negative dispersion coefficient. We evaluate two types of laser, with different characteristics and we determine optimal optical output power in every case. We have demonstrated that enhanced system performance, which uses a positive dispersion fiber, can be achieved if positive chromatic dispersion in the optical fiber is equalized by SPM, whereas laser transient chirp can be compensated using a negative dispersion fiber.

\section{Dispersion in optical fibers}

Dispersion occurs when a wave interacts with a medium or passes through an inhomogeneous geometry. It causes pulses to broaden in optical fibers, degrading signals over long distances. 
If dispersion is too high, a group of pulses representing a bit-stream will spread in time making the bit-stream unintelligible. This limits the length of link or the information capacity of the fiber without regeneration. There are different types of dispersion, which all involve the dependence of the phase velocity or phase delay of light in some medium: intermodal dispersion, polarization mode dispersion and chromatic dispersion.

Intermodal dispersion results from different propagation characteristics of higher-order transverse modes in waveguides and can limit the possible data rate of a system for optical fiber communications based on multimode fibers.

Polarization mode dispersion results from polarization-dependent propagation characteristics. In a waveguide, different polarizations of light travel at different speeds due to imperfections and asymmetries, causing spreading of optical pulses.

Chromatic dispersion is the result of the wavelength dependence of the group velocity $\mathrm{v}_{\mathrm{g}}$.

The most commonly used chromatic dispersion parameter is $\mathrm{D}$, defined as

$$
D=\frac{d}{d \lambda}\left(\frac{1}{v_{g}}\right)=-\frac{2 \pi c}{\lambda^{2}} \beta_{2}
$$

Where $\mathrm{c}$ is the velocity of light in vacuum, $\lambda$ is the wavelength and $\beta_{2}$ is the Group Velocity Dispersion parameter (GVD).

The reason for defining the dispersion in this way is that $|\mathrm{D}|$ is the temporal pulse spreading $\Delta \mathrm{t}$ per unit bandwidth $\Delta \lambda$ per unit distance travelled, commonly reported in $\mathrm{ps} / \mathrm{nm} \mathrm{km}$ form optical fibers. $\mathrm{D}$ is generally used to indicate the amount of dispersion in fiber specifications. If $\mathrm{D}$ is less than zero, the medium is said to have positive dispersion and if $\mathrm{D}$ is greater than zero, the medium has negative dispersion.

There are two sources of chromatic dispersion: material dispersion and waveguide dispersion.

Material dispersion occurs because an optical pulse emitted by a light source has a certain spectral width. The index of refraction of a material is dependent on the wavelength, so each frequency component travels at a slightly different speed. As the distance increases, the pulse becomes broader as a result. Waveguide dispersion refers to differences in the signal speed depending on the distribution of the optical power over the core and cladding in an optical fiber.

Material dispersion and waveguide dispersion have opposite effects (see Figure 1) and due to the difference of signs, there is a lambda $\left(\lambda_{\mathrm{ZD}}\right)$ for which the chromatic dispersion coefficient is zero.

Fiber manufacturers can manipulate these effects to change the location and slope of the chromatic dispersion curve so it is possible to shift the zero dispersion wavelength to the 1.5- $\mu \mathrm{m}$ region ( $\mathrm{C}$ band).

All fibers with $\lambda_{Z D}$ near to $1550 \mathrm{~nm}$ are called dispersion-shifted Fibers (DSF) and fibers with $\lambda_{Z D}$ outside $C$ band are called non-zero dispersion shifted (NZ-DSF). Figure 2 shows the dispersion curves for different models of optical fiber. 


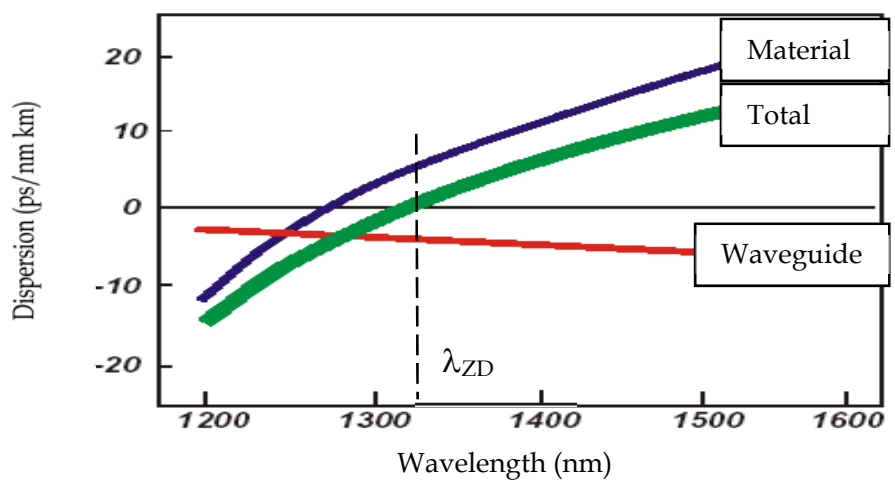

Fig. 1. Chromatic dispersion curve

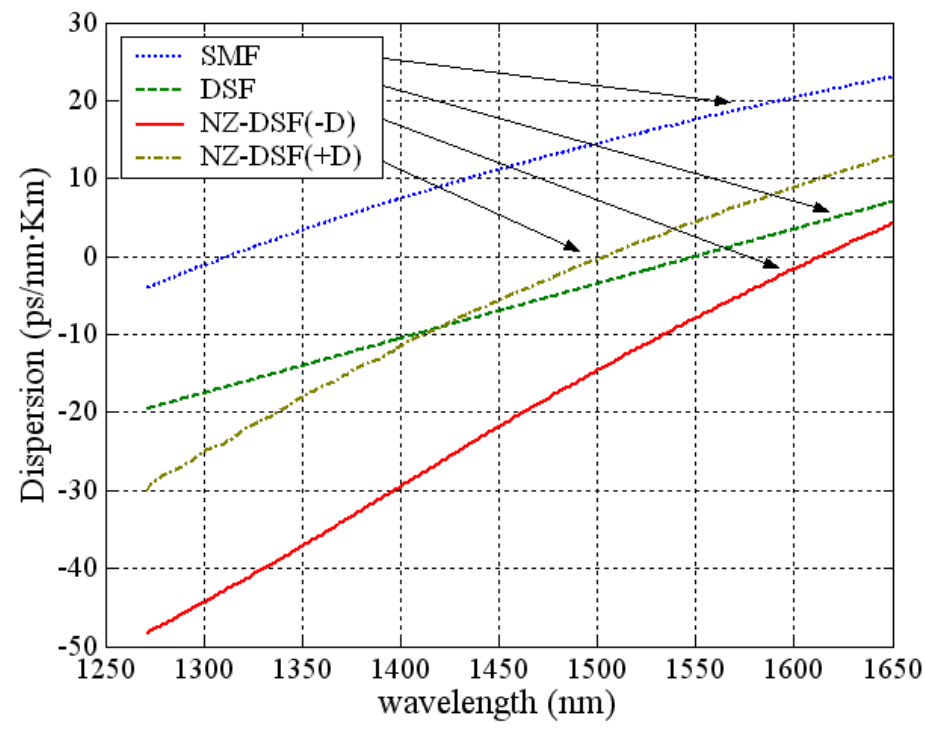

Fig. 2. Dispersion coefficients for different types of fibers.

For wavelengths longer than the zero dispersion wavelength $\left(\lambda_{\mathrm{ZD}}\right)$, called anomalous dispersion region, the rate of change of the index of refraction with respect to the wavelength changes sign, in which case high frequency components travel faster than the lower ones, and the pulse becomes negatively chirped or down-chirped, decreasing in frequency with time.

By the other hand, if a light pulse is propagated through a normally dispersive medium (for wavelengths no longer than the zero dispersion wavelength) the result is the higher frequency components travel slower than the lower frequency components. The pulse therefore becomes positively chirped or up-chirped, increasing in frequency with time. 


\section{The effect of dispersion on directly modulated systems}

Currently DML transmitters are used to build low-cost optical communication architectures.

A block diagram of the externally modulated transmitter is illustrated in Fig. 3, in contrast with directly modulated transmitter, in Figure 4 . It consists of a bit random generator, which determines the sequence of bits, $a_{k}$, that will be sent to an electric pulse generator, with NRZ format, which injects a modulation current, $I(t)$ to a Mach-Zehnder modulator. The CW Laser output port is connected to the Mach-Zehnder Modulator.

In both cases, the injected laser current is given by the expression:

$$
I(t)=I_{b}+\sum_{k=-\infty}^{\infty} a_{k} I_{p}(t-k T)
$$

where $I_{b}$ is the bias current, $T$ is the period of the modulation pulse, the sequence of bits transmitted $\left(a_{k}=1(0)\right.$ if a binary one (zero) is transmitted during the $k_{t h}$ time), and $I_{p}(t)$ is the applied current pulse.

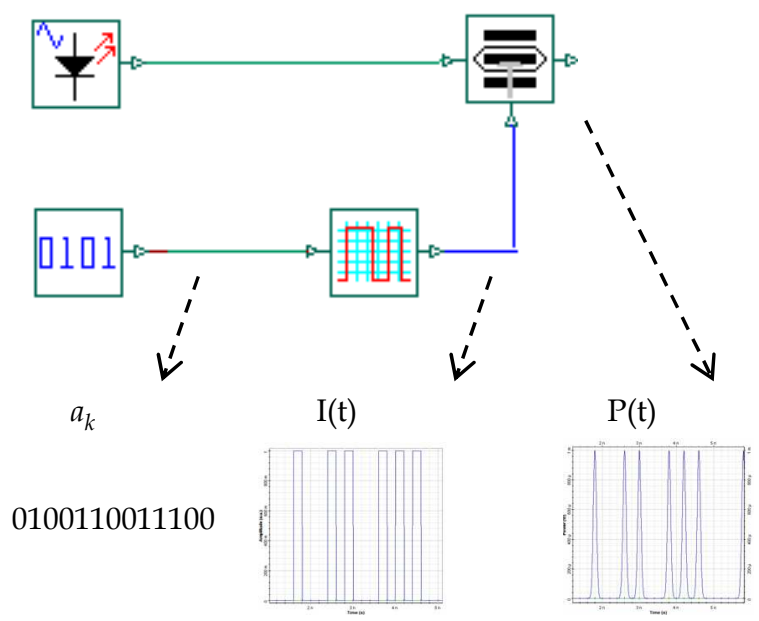

Fig. 3. Block diagram of the externally modulated transmitter

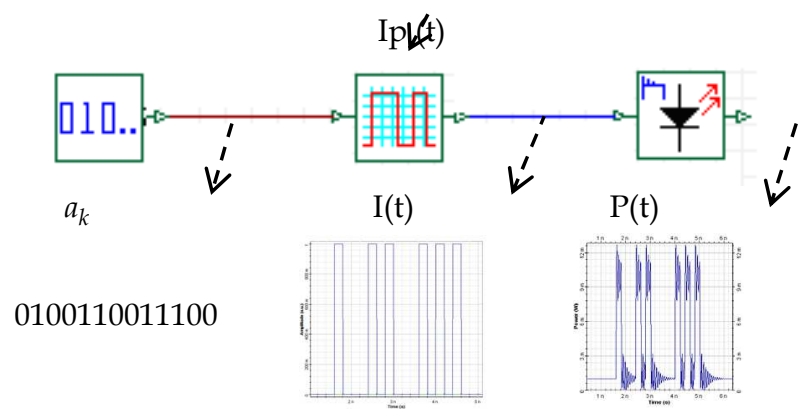

Fig. 4. Block diagram of the directly modulated transmitter 
In an externally modulated laser, the optical power output pulse of the laser, $\mathrm{P}(\mathrm{t})$, is given by

$$
P(t)=\eta_{0} \cdot \frac{h v}{q} \cdot \sum_{k=-\infty}^{+\infty} a_{k} I_{p}(t-k T)
$$

where $\eta_{0}$ is differential quantum efficiency of the laser, hv the photon energy at the optical frequency $v, q$ is the charge of an electron and $I p(t)$ the applied current pulse.

However, expression (3) is not applicable in case of directly modulated sources where the injected current that modulates the laser introduces a shift in the emission frequency (chirp frequency). As a consequence, the optical power output pulse is not a linear transformation of the applied current pulse.

The optical power and chirping response of the semiconductor laser to the current waveform I $(t)$ is determined by means of the large-signal rate equations [Tomkos 2001b], which describe the interrelationship of the photon density, carrier density, and optical phase $\phi(t)$, within the laser cavity.

Thus, the emission frequency associated with the output of the laser (chirp) can be formulated by:

$$
\Delta v(t)=\frac{1}{2 \pi} \frac{d \phi}{d t}
$$

where $\phi$ is the optical phase, which variation with the time depends on

$$
\frac{d \phi}{d t}=\frac{1}{2} \alpha\left[\Gamma v_{g} a_{0}\left(n-n_{t}\right)-\frac{1}{\tau_{p}}\right]
$$

$\mathrm{n}_{\mathrm{t}}$ represents the carrier density at transparency, $\alpha$ the line-width factor or Henry's factor, $\Gamma$ the confinement factor, $v g$ the group velocity, $\mathrm{a}_{0}$ the gain coefficient, and $\tau_{\mathrm{p}}$ is the photon lifetime.

To analyze the interaction of dispersion with the chirp generated in directly modulated systems we initially study systems where pulse have got Gaussian shapes and finally pulses with others forms.

\subsection{Gaussian pulses}

Even though the shape of optical pulses representing 1 bits in a bit stream is not necessarily Gaussian, one can gain considerable insight into the effects of fiber dispersion by focusing on the case of a chirped Gaussian pulse with the input field.

In Fig. 5 the pulse chirp and optical phase, is plotted together with the pulse intensity. As it can be seen quadratic changes in the optical phase corresponds to linear frequency variations, for this reason, such pulses are said to be linearly chirped [Agrawal 2010].

In a linear chirp, the instantaneous frequency $f(t)$ varies linearly with time:

$$
f(t)=f_{0}+C t
$$




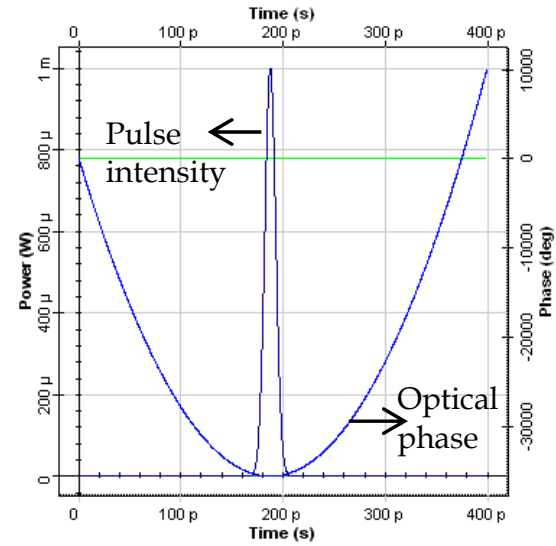

(a) Pulse intensity and optical phase

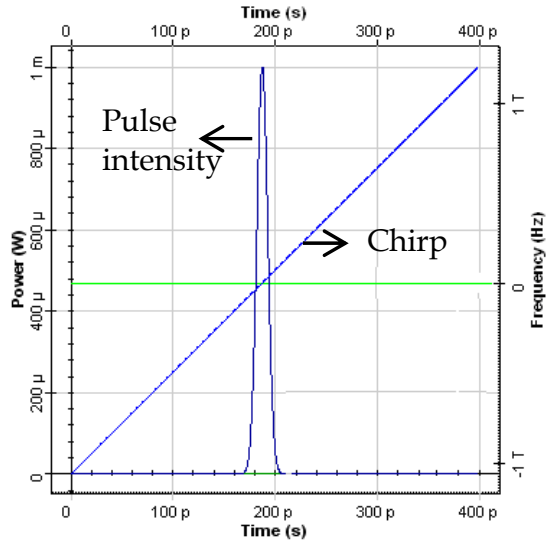

(b) Pulse intensity and chirp

Fig. 5. Pulse intensity, optical phase and chirp in an optical Gaussian pulse.

where $f_{0}$ is the starting frequency (at time $t=0$ ) and $C$ is the rate of frequency increase or chirp rate. In a chirp-free Gaussian pulse, the optical frequency remains constant throughout the pulse, as shown in Figure 6a, whereas an up-chirp (down-chirp) pulse means that the instantaneous frequency rises (decreases) with time (Figures $6 \mathrm{~b}$ and $6 \mathrm{c}$ ).
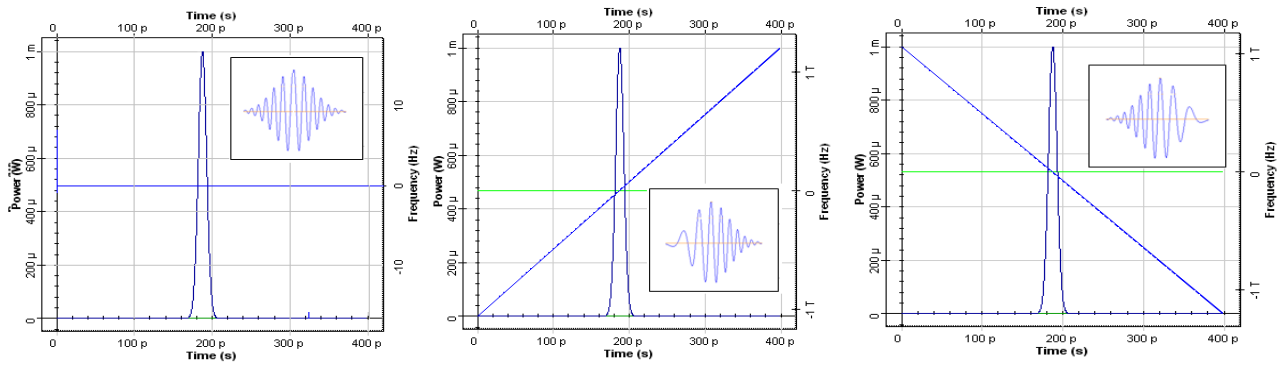

(a) Chirp-free gaussian pulse

(b) Up-chirped pulse

(c) Down-chirped pulse

Fig. 6. Pulse intensity, optical phase and chirp for Gaussian opical pulses.

\subsection{Non Gaussians optical pulses}

In directly modulated sources, optical pulses are often non-Gaussian and may exhibit considerable chirp caused by carrier-induced changes in the mode index. 
In these cases, the chirp is not linear and therefore can not be characterized by the value of the chirp rate constant $(\mathrm{C})$ and it can be calculated from the following expression [Coldren 1995]

$$
\Delta v(t)=\frac{\alpha}{4 \pi}\left(\frac{1}{P(t)} \frac{d P(t)}{d t}+\left[\frac{P(t) \varepsilon}{\tau_{p}}-\frac{\beta \Gamma n(t)}{\tau_{n} P(t)}\right]\right)
$$

This equation evaluates the chirp for Fabry-Perot semiconductor laser, but is used as a good approximation for DFB lasers [Morgado 2003]. By the other hand, this expression can be simplified whenever working with modulation frequencies above $100 \mathrm{MHz}$, then comes to a new equation that depends on two laser intrinsic parameters: the adiabatic chirp coefficient (к) and the Henry factor ( $a)$.

$$
\Delta v(t)=\frac{\alpha}{4 \pi}\left(\frac{1}{P(t)} \frac{d P(t)}{d t}+\frac{P(t) \varepsilon}{\tau_{p}}\right)=\frac{\alpha}{4 \pi}\left(\frac{1}{P(t)} \frac{d P(t)}{d t}+\kappa P(t)\right)
$$

The adiabatic coefficient, $\mathrm{k}$, depends on the laser structure, being the figure that takes into account the output power and the generated chirp. The relationship to the photon energy, $h v$, optical frequency, $v$, laser quantum efficiency, $\eta_{0}$, confinement factor, $\Gamma$, cavity volume, $\mathrm{V}_{0}$ and gain compression factor, $\varepsilon$, is in agreement with the expression:

$$
\kappa=\frac{2 \Gamma}{\eta_{0} h \nu V_{a}} \varepsilon
$$

Its units of measurement are (W S) $)^{-1}$ and takes values around $10^{12}$.

Henry factor, normally called linewidth enhancement factor, quantify the effect of the change of refractive index with carrier density on the dynamical properties of semiconductor lasers, [Henry 98]

$$
\alpha=-\frac{\partial \chi_{r} / \partial N}{\partial \chi_{i} / \partial N}=-\frac{4 \pi}{\lambda} \frac{d n}{d g}
$$

where $\mathrm{n}$ is the refractive index, $\mathrm{g}$ is the gain per unit length, $\lambda$ is the wavelength and $\mathrm{N}$ is the carrier density.

A lot of experiments have reported a great variety of values for the $a$-factor ranging from 0 to values of the order of those commonly observed in bulk and quantum well materials (1.53). Larger values for the $a$-factor than those published for quantum well devices have also been reported and a value for $\alpha$ as high as 60 has been published.

The first term of (8), called transient chirp, causes variations in the pulse width and is scaled by a. The second term (adiabatic chirp) produces a frequency shift proportional to the instantaneous optical power, and scaled, in addition to $a$, by parameter $\mathrm{K}$ [C.del Rio 2008]. Figure 7 shown two types of chirp in DML. We can observe a transient chirp corresponds to the chirp that occurs during the transition between steady states and an adiabatic chirp corresponds to the frequency offset between steady state output powers of the laser. 


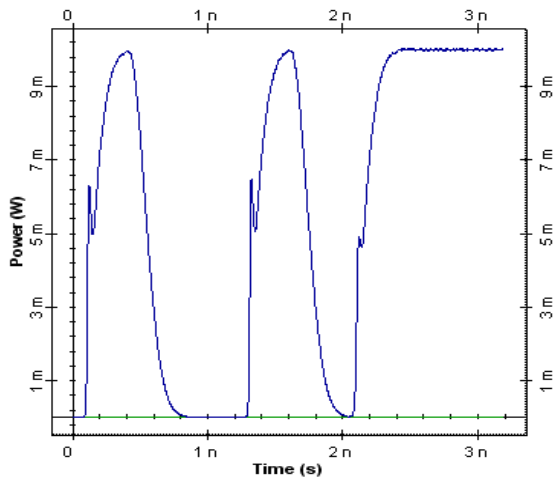

(a) Optical pulse

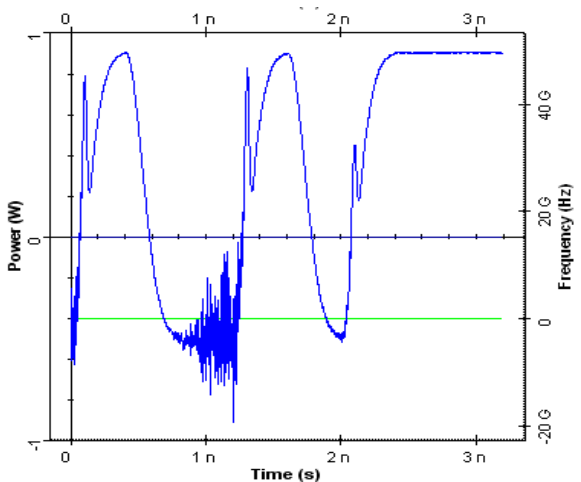

(b) Adiabatic chirp DML-A

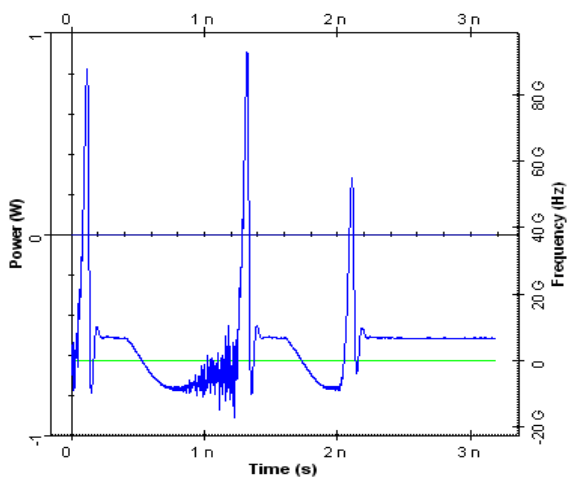

(c) Transient chirp DML-T

Fig. 7. Optical pulses shapes (a) and chirp in an adiabatic (b) and transient (c) dominated laser.

Depending on the term in equation 8 that predominate over the other DMLs can be classified as transient (DML-T) or adiabatic (DML-A) chirp dominated. This terminology was first used the Hinton by 1993 [Hinton 93]. 
Each extreme category has significantly different optical spectra. DML-T lasers have higher bandwidths to DML-A, as shown in Figure 8.

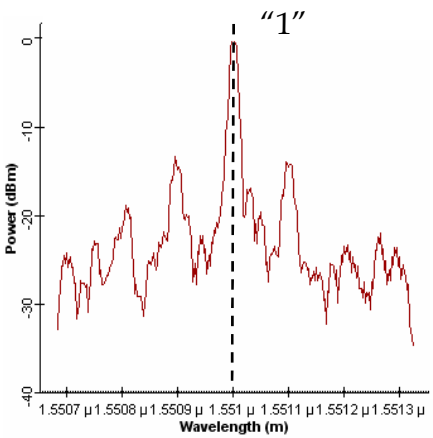

(a) DML-T

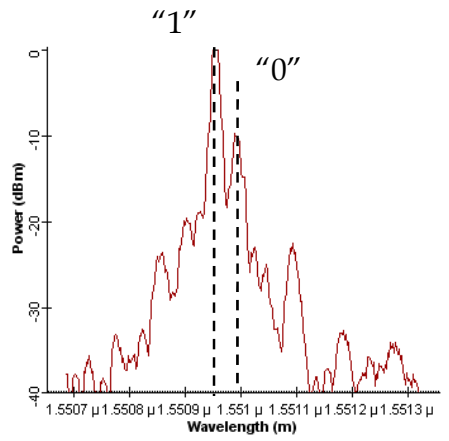

(b) DML-A

Fig. 8. Spectrum of transient chirp dominated (a) and adiabatic chirp dominate laser (b)

Besides the difference in the spectral width, is observed in lasers DML-T that the emission peak is centered at the nominal frequency of emission, which corresponds to the peak frequency during continuous vawe transmission (Figure 8a). However, the laser DML-A there are two different frequency peaks corresponding to carrier frequencies of the bits " 1 " and "0" (Figure $8 \mathrm{~b}$ ). The separation between the wavelengths of the carriers of bits 0 and 1 is directly related to the adiabatic chirp of the signal and, therefore, depends on the output power.

Figure 9 represents the chirp of bit "1" when the output power varies from 0.1 to $15 \mathrm{~mW}$. As can be seen, the higher the laser emission power the higher the adiabatic chirp generated. In this particular case, the peak-to-peak chirp generated when the value of output power is 0.1 $\mathrm{mW}$ is $8 \mathrm{GHz}$ and $35 \mathrm{GHz}$ if the power change to $15 \mathrm{~mW}$.

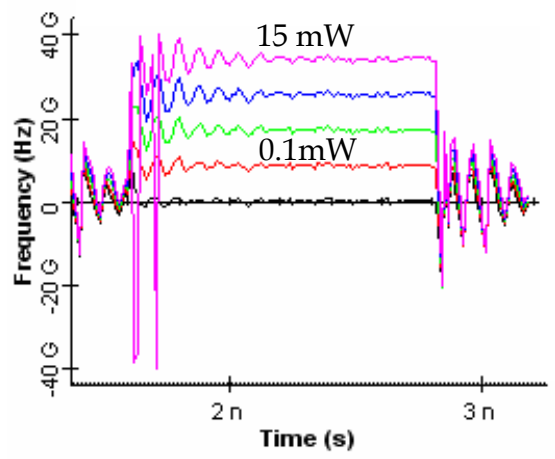

(a) Chirp

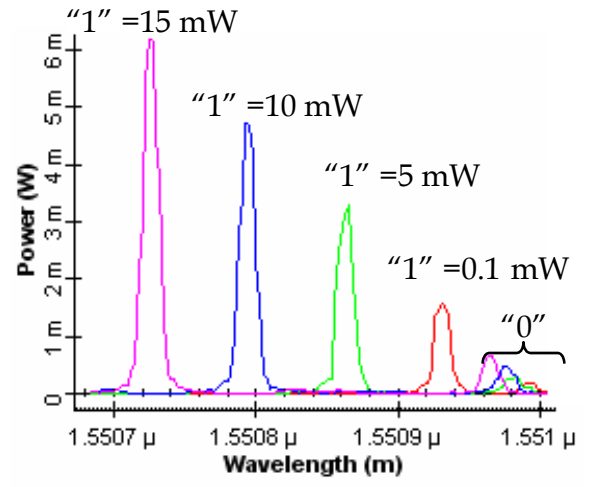

(b) Spectrum

Fig. 9. Chirp (a) and spectrum (b) for 10 output power DML for 0.1 to $15 \mathrm{~mW}$

The frequency chirp, due to the adiabatic chirp and shown in Figure 9a, implies a shift in the wavelength emission, as it shown in Figure 9b. This optical frequency shift from the actual laser output frequency is the principal cause of system performance limitations. 


\subsection{Interaction dispersion-chirp-SPM}

Gaussian pulse remains Gaussian during propagation in a transparent medium but its chirp and with change due to the effects of chromatic dispersion and nonlinearities (e.g. self-phase modulation arising from the Kerr effect).

Therefore, when a pulse with an initial chirp C, crosses a distance $\xi$ in a dispersive media, the chirp will acquire a new value given by $C_{1}$ and the pulse will be broadened by actor represented by bf

$$
\begin{gathered}
\text { bf }(\xi)=\left[(1+\mathrm{sC} \xi)^{2+} \xi\right]^{1 / 2} \\
\mathrm{C}_{1}(\xi)=\mathrm{C}+\mathrm{s}\left(1+\mathrm{C}^{2}\right) \xi
\end{gathered}
$$

Where $s$ is the sign of $\beta_{2}(+1$ if the pulse propagates in the normal dispersion and -1 in the anomalous dispersion) and $\xi$ is related to $\beta_{2}$ and FWHM (full width at half-maximum).

Namely, the shape of the signal at the output of the optical fiber is affected by the sign of both $\beta_{2}$ (fiber parameter) and $C$ (transmitter parameter).

An unchirped pulse $(C=0)$ broadens monotonically by a factor of $\left(1+\xi^{2}\right)^{1 / 2}$ and develops a negative chirp across the fiber. Chirped pulses, on the other hand, may expand or compress depending on whether $\beta_{2}$ and $C$ have the same or contrary signs.

When $\beta_{2} \mathrm{C}>0$, a chirped Gaussian pulse broadens monotonically at a rate faster than that the unchirped pulse. The reason is related to the fact that the dispersion-induced chirp adds to the input chirp because the two contributions have the same sign. The situation changes for $\beta_{2} \mathrm{C}<0$. In this case, the contribution of the dispersion-induced chirp is of a kind opposite to that of the input chirp.

In Figure 10, the pulse chirp is plotted together with the pulse intensity. Initial narrowing of the pulse for the case $\beta_{2} \mathrm{C}<0$ can be explained by noticing that the faster frequency components are in the trailing edge, and the slower in the leading edge of the pulse. As the pulse propagates, the faster components will overtake the slower ones, leading to pulse narrowing. At $\mathrm{L}=\mathrm{L}_{2}$ full compensation between dispersion induced chirp and the initial one

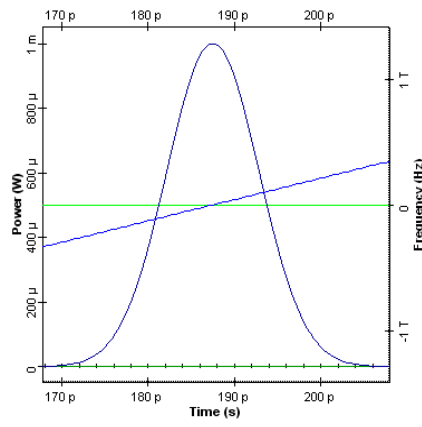

(a) $\mathrm{L}_{1}$

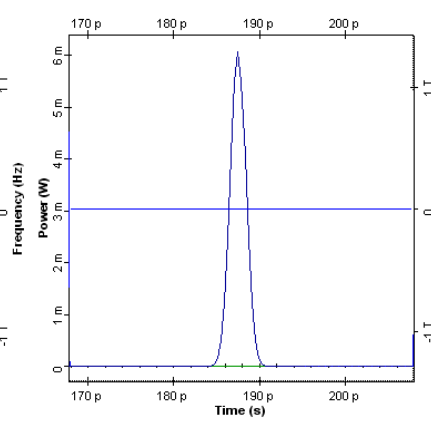

(b) $\mathrm{L}_{2}$

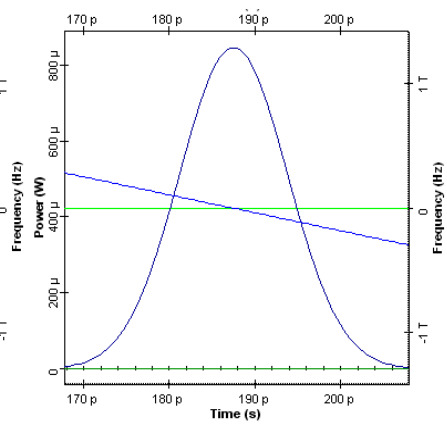

(c) $\mathrm{L}_{3}$

Fig. 10. The output optical pulse signal for the different transmission length $\left(\mathrm{L}_{1}, \mathrm{~L}_{2}\right.$ and $\left.\mathrm{L}_{3}\right)$ 
will occur. At this length $\mathrm{L}_{2}=|\mathrm{C}| /\left(1+\mathrm{C}^{2}\right), \mathrm{C}_{1}$ becomes zero and the pulse becomes unchirped. We define this situation as optimal.

Finally, with further propagation, the fast and the slow frequency components will tend to separate in time from each other and pulse broadening will be observed.

On the other hand, the SPM alone leads to pulse chirping, with the sign of the SPM-induced chirp being opposite to that induced by anomalous GVD.

In Figure 11, the leading edge of the pulse becomes red-shifted and the trailing edge of the pulse becomes blue-shifted. If the effects of anomalous dispersion were present, with the chirp induced by SPM some pulse narrowing would occur. This means that the effect of SPM counteracts GVD.
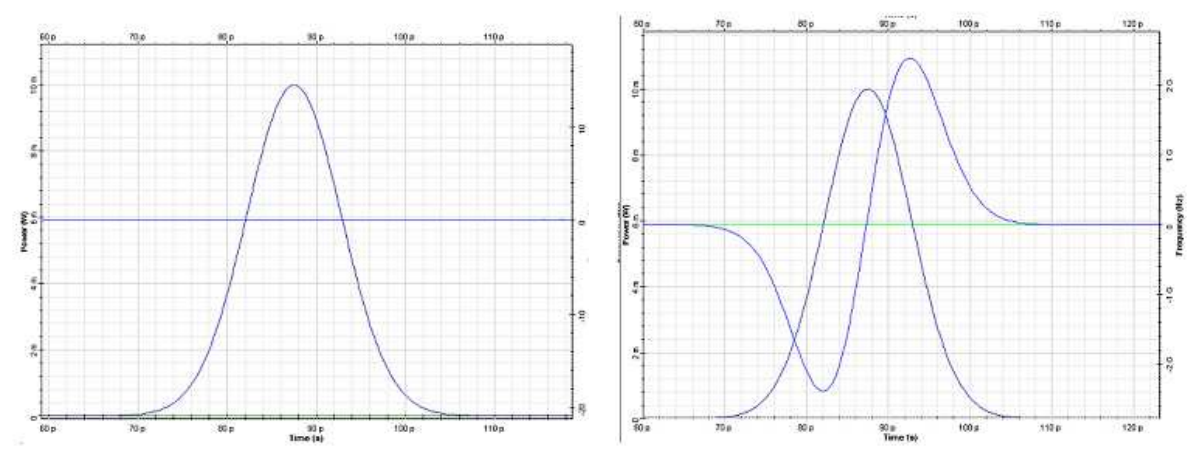

Fig. 11. Input (left) and output (right) pulse shape and chirp.

The effect of GVD on the pulse propagation depends, mainly, on whether or not the pulse is chirped, the laser injection pulse shape, [del Rio 2010], and also on the fiber SPM (Self Phase Modulation [Hamza, M. Y., Tariq, S. \& Chen, L. 2006, 2008]. With the correct relation between the initial chirp and the GVD parameters, the pulse broadening (which occurs in the absence of any initial chirp) will be preceded by a narrowing stage (pulse compression). On the other hand, the SPM alone leads to a pulse chirping, with the sign of the SPMinduced chirp, being opposite to that induced by anomalous GVD. This means that in the presence of SPM, the GVD induced pulse-broadening will be reduced (in the case of anomalous), while extra broadening occurs in the case of normal GVD.

\section{Enhancing the performance of systems using negative and positive dispersion fibers}

In this section, we study that the transmission performance depends strongly on dispersion fiber and DML output power. We demonstrated that systems using SMF fibers can achieve a good performance if the DML output power is properly chosen. Finally, we have found a mathematical expression that make an estimation for a power value to fix the laser power output for each channel in WDM systems.

In order to study the CWDM system performance a simple arrangement is proposed, as can be seen in Figure 12. We have selected 16 output channels with wavelengths, in agreement 
with Recommendation ITU-T G. 694.2. The pulse pattern was a periodic 128-bit OC-48 (2.5 $\mathrm{Gb} / \mathrm{s}$ ) nonreturn-to-zero (NRZ). After transmission through $100 \mathrm{~km}$ of fiber, channels are demultiplexed and detected using a typical pin photodiode.

We have used two kinds of optical fibers; the already laid and widely deployed single-mode ITU-T G.652 fiber (SMF) and the ITUT-T G.655 fiber with a negative dispersion sign around $\mathrm{C}$ band (NZ-DSF). It is well known, SMF fiber dispersion coefficient is positive in the whole telecommunication band from O-band to L-band and the dispersion coefficient of the NZDSF fiber is negative in the optical frequency range considered. For our purpose, the same spectral attenuation coefficient of both fibers has been considered whose water peak at 1.38 $\mu \mathrm{m}$ is well suppressed. The dispersion slope, effective area and nonlinear index of refraction are compliant with typical conventional G.652 and G.655 fibers.
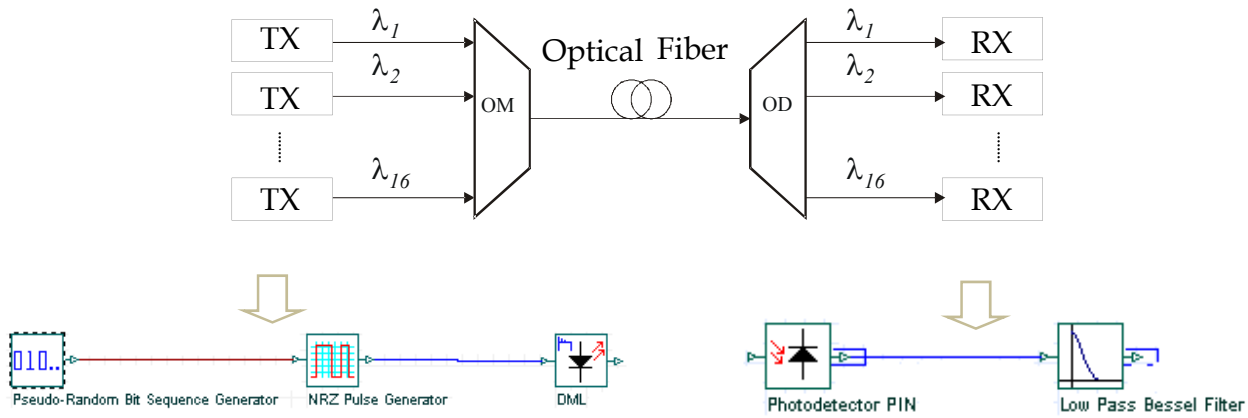

Fig. 12. Arrangement set up of simulated transmission link.

We have to point out that the transmission performance of waveforms produced by directly modulated lasers in fibers with different signs of dispersion, depends strongly on the characteristics of the laser frequency chirp. For this reason, we have modeled two DMLs (made up of DFB-DMLs), by using the Laser Rate Equations in agreement with that reported in [Tomkos 2001b], both DMLs presenting extreme behaviors [Hinton 1993]: DML-A is strongly adiabatic chirp dominated; $\alpha=2.2$ and $\mathrm{k}=28.7{ }^{*} 10^{12}(\mathrm{~W} . \mathrm{s})^{-1}$ and DML-T is strongly transient chirp dominated; $\alpha=5.6$ and $\mathrm{k}=1.5{ }^{*} 10^{12}$ (W.s) ${ }^{-1}$. The $\alpha$ and $\mathrm{k}$ values used in our simulation are in agreement with potential commercial devices [Osinki 1987, Peral 1998, Rodríguez 1995].

In this work, we are mainly interested in comparing the system performance based on the type of fiber and DML used; for this reason, the rest of link components have been modeled by considering ideal behavior.

The performance of transmission systems is often characterized by the bit error rate (BER), which is required to be smaller than approximately $10^{-12}$ for most installed systems. Experimental characterization of such systems is not easy since the direct measurement of BER takes considerable time at these low BER values. Another way of estimating the BER is using the $\mathrm{Q}$ of the system, which can be more easily modeled than the BER.

The parameter $Q$, the signal-to-noise ratio at the decision circuit in voltage or current units, is given by the expression[Alexandre 1997] 


$$
Q=\frac{I_{1}-I_{0}}{\sigma_{1}+\sigma_{0}}
$$

where $I_{i}$ and $\sigma_{i}$ are average values and variances of the " 1 " and " 0 " values for each pattern. $Q$ factor can be considered just a qualitative indicator of the actual BER and it can expressed as

$$
B E R=\frac{1}{2} \operatorname{erfc}\left(\frac{Q}{\sqrt{2}}\right)
$$

This parameter guarantee an error-free transmission of Q-Factor higher than 7, corresponding to a BER lower than 10-12 .

In order to study the transmission performance of DMLs presenting extreme behavior on a fiber with positive or negative dispersion, a set of simulations were carried out; called Cases A, B, C and D, as shown in Table 1. The quality of transmission between them has been compared. Thus, Case-A deploys DML-A lasers and SMF fiber, Case-B: DML-A/NZ-DSF, Case-C: DML-T/SMF and Case-D: DML-T/ DSF.

\begin{tabular}{|c|c|c|}
\hline Case & DML & Optical Fiber \\
\hline A & DML-A & SMF \\
\hline B & DML-A & NZ-DSF \\
\hline C & DML-T & SMF \\
\hline D & DML-T & NZ-DSF \\
\hline
\end{tabular}

Table 1. Different configurations for the simulated system

The DML output power of all channels was varied from $-10 \mathrm{dBm}$ to $10 \mathrm{dBm}(0.1-10 \mathrm{mw})$, and the performance, in terms of $Q$-Factor, is analyzed for each transmitted channel.

Figure 13 shows the Q-Factor dependence on channel power for the wavelength channel centered at $1551 \mathrm{~nm}$.

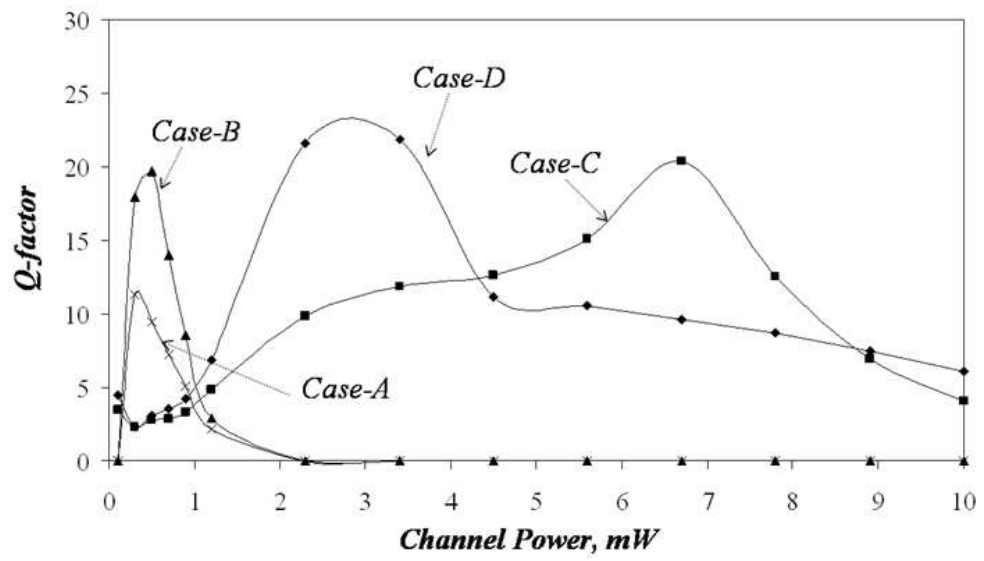

Fig. 13. Simulated results for the transmission performance, Q-Factor, at $1551 \mathrm{~nm}$ wavelength after transmission over $100 \mathrm{~km}$ of positive and negative dispersion fiber 
Independently of the Case and wavelength channels, the Q-Factor always presents a maximum value for a specific DML output power [Horche 2008]. This behaviour demonstrates the existence of an optimum channel power that will have to be considered during the system design.

This optimum value corresponds with the power value that allows compensating the laser chirp with the fiber dispersion and it depends on the combination of components used in each case.

\section{1 $A$ and B cases: Adiabatic dominated laser}

A and B Cases use adiabatic chirp dominated DML-A lasers. The Qmax value is reached at 0.3-0.46 $\mathrm{mw}$, independently of the fiber type. Over this value the function drastically gets worse when increasing the output laser power. In both cases the type of the laser used in the simulation is an adiabatic chirp dominated, so for values over $0.4 \mathrm{~mW}$ the filter reduces partially the spectrum and this phenomenon closes the eye diagram.

Fig. 14 shows the spectrum of adiabatic chirp dominated laser together with the transfer function of a Gaussian filter. The shift of the spectrum towards blue would cause a bigger reduction of the peak emission of bit " 1 " than the one produced on the peak of bit " 0 ". This would bring both " 1 " and " 0 peak emission power closer and the eye diagram be closed.

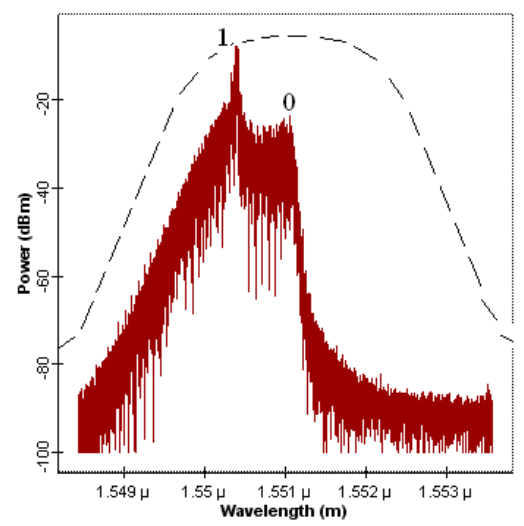

Fig. 14. Spectrum of adiabatic chirp dominated laser together with the transfer function of a Gaussian filter

On the other hand, the power waveform coming from DML suffers a deformation when getting through the dispersive media. In the case of DML-A, the result of the interplay of the dispersion with the specific chirp characteristics will result in a high intensity spike at the front of the pulses for transmission through a fiber with positive dispersion (SMF) and at the end for negative dispersion (NZ-DSF) [Krehlik06], as can be seen at the top of the Figure 15.

The absolute value of the dispersion (and not its sign) will play a major role in the transmission performance. Thus, the performance corresponding to transmission through an SMF fiber will be worse than that corresponding to transmission through an NZ-DSF fiber because of the larger absolute value of the dispersion. 
Figure 15 represents the power waveforms for five different optical output powers (from 0.5 to $4 \mathrm{mw}$ ) after transmission through $100 \mathrm{~km} \mathrm{NZ-DSF} \mathrm{fiber.}$

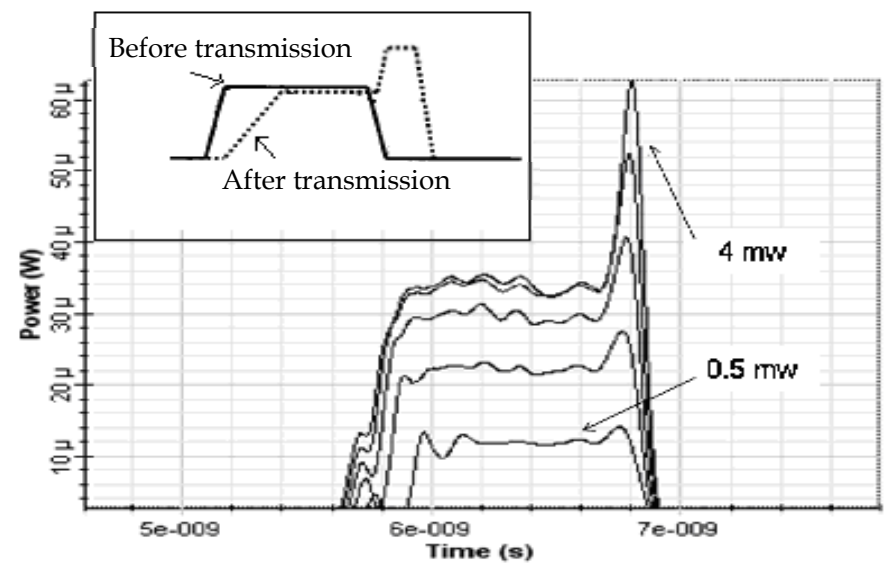

Fig. 15. Shapes of optical pulses for different DML-A output powers, after transmission through $100 \mathrm{~km}$ negative dispersion fiber

The increment of $P_{c h}$ will result in a higher intensity spike at the trailing edge of the pulse. As consequence the eye pattern after transmission will be severely closed.

In Fig. 16 the eye diagrams are shown for the case of the adiabatic chirp dominated transmitter after transmission over $100 \mathrm{~km}$ of a negative dispersion fiber for (a) $\mathrm{P}_{\mathrm{ch}}=0.46$ $\mathrm{mw}$ (optimum power) and (b) $\mathrm{P}_{\mathrm{ch}}=1 \mathrm{mw}$. For $\mathrm{P}_{\mathrm{ch}}=0.46 \mathrm{mw}$, the eye pattern is clearly open, while for $\mathrm{P}_{\mathrm{ch}}=1 \mathrm{mw}$ eye pattern experiencing more than $3 \mathrm{~dB}$ eye closure.

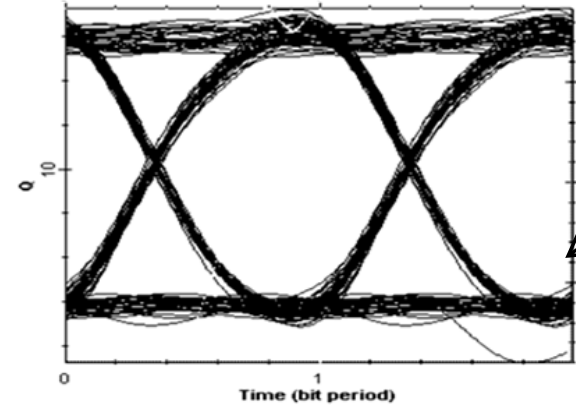

a) $\mathrm{P}_{\mathrm{ch}}=0.5 \mathrm{mw}$

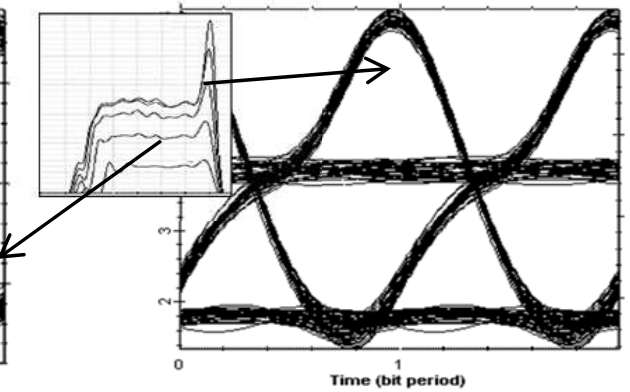

(b) $\mathrm{P}_{\mathrm{ch}}=1 \mathrm{mw}$

Fig. 16. Eye diagrams for the case of the adiabatic chirp dominated transmitter after transmission over $100 \mathrm{~km}$ of a negative dispersion fiber for (a) $P_{c h}=0.46 \mathrm{mw}$ and (b) $P_{c h}=1$ mw.

For small powers, the $Q$-Factor increases with $\mathrm{P}_{\mathrm{ch}}$ because a large amount of power reaches the detector. For higher $P_{c h}$ the optical pulse deformation arising from chirp induced by DML becomes too large and causes an error in pulse reconstruction. 


\section{2 $C$ and $D$ cases: Transient dominated laser}

$\mathrm{C}$ and D Cases use transient chirp dominated DML-T lasers. For Case-C (DML-T/SMF), the $\mathrm{Q}_{\max }$ value takes place for an output power of $6.7 \mathrm{mw}$ approximately. In Case-D (DML$\mathrm{T} / \mathrm{DSF}$ ), the necessary output power to reach the $\mathrm{Q}_{\max }$ is around 2.3-3.4 $\mathrm{mw}$.

In DML-T, the wavelength shift by laser transient chirp is a blue shift during the pulse rise time and a red shift during the pulse fall time; exactly the opposite effects takes place with SPM (Self-phase-modulation) [Suzuki 1993]. Therefore, the optical pulse chirped by direct modulation is compressed in fibers with negative dispersion (NZ-DSF), while that chirped by SPM is compressed in fibers with positive dispersion (SMF).

As it can be seen in Figure 13, for channel power Pch from 0.1 to $4 \mathrm{mw}$, the performance of system that uses an NZ-DSF fiber (D-Case) is better than that of an SMF fiber (C-Case). In this power range, SPM magnitude is not enough and the wavelength shift by laser transient chirp is the predominant effect. Thus, the optical pulse chirped by direct modulation is compressed in fibers with negative dispersion (NZ-DSF) and uncompressed in fibers with positive dispersion (SMF). Therefore, case $\mathrm{D}$ is better than case $\mathrm{C}$, however, for $\mathrm{P}_{\mathrm{ch}}$. from 4 $\mathrm{mw}$ to $9 \mathrm{mw}$, Case-C (DML-T/SMF) presents a better performance than Case D (DMLT/NZ-DSF) because of the increment in the magnitude of the SPM in the optical fiber and, therefore, chromatic dispersion of the positive dispersion fiber is equalized by the SPM as long as the pulses are broadened for negative dispersion fiber. As resulting from this, the eye pattern after the transmission through SMF fiber will be more open than using NZ-DSF fiber when higher output power is used.

Figure 17 shows the eye diagram of Case-C (a) and Case-D (b). In both cases a $P_{c h}$ of $7 \mathrm{mw}$ was used and the eye diagram is measured for the signal transmission after $100 \mathrm{~km}$ of dispersion fiber at $1551 \mathrm{~nm}$ wavelength. After the transmission through SMF, the eye look perfectly open (Fig 17a) while the eye pattern after transmission through NZ-DSF is severely closed (see Fig 17b) and intersymbol interference will occur. On other hand, the different dispersion sign will only affect the asymmetry of the eye diagram, as is obvious from the results of Fig. 17.

Therefore, we can conclude that systems using an SMF fiber can have a similar or better performance to those systems that use an NZ-DSF fiber if the DML is transient chirp dominated and its output power is properly chosen.

\section{Management of the power channel of to enhance CWDM system performance}

In order to analyze the influence of the selected wavelength in a CWDM system, simulations varying the number of channels from 1 to 16 have been carried out, using the same schematic arrangement set up shown in Fig. 12. The channel wavelengths were between 1531 and $1591 \mathrm{~nm}$. In this case, this wavelength range was used due to the system does not need optical amplifiers. Some channels were located at compatibles frequencies with CWDM ITU-T grid in order to, in the future, extend this work to whole useful fiber optic spectral range (1271-1611 $\mathrm{nm})$.

In every case, the $Q$-Factor shows a maximum value for a given optical output power. In $\mathrm{A}$ and B Cases, due to small powers of channels, $Q_{\max }$ is almost independent of number of 


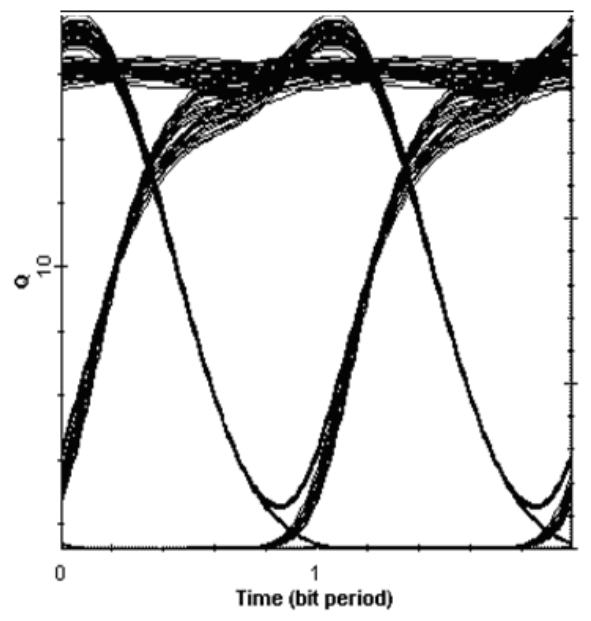

(a) Positive

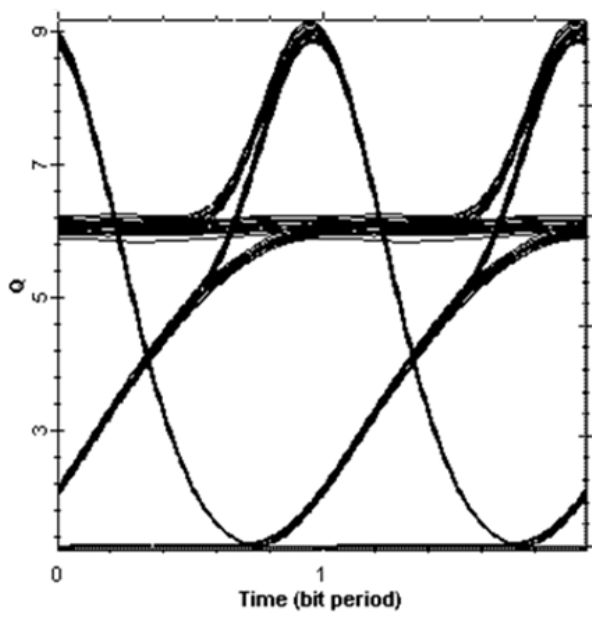

(b) Negative

Fig. 17. Eye diagram at the receiver side of a $2.5 \mathrm{~Gb} / \mathrm{s}$ transient chirp dominated transmitter (7 mw of output power at $1551 \mathrm{~nm}$ wavelength) over (a) SMF fiber where the dispersion is positive and (b) NZ-DSF fiber where the dispersion is negative

channels. In C and D Cases, this maximum value decreases with the increment of the number of channels used manly due to crosstalk between channels and others no-lineal effects. However, the $Q_{\max }$ value, for a given channel, takes place for a very similar output power.

Figure 18 shows the $Q$-Factor versus channel power for channels centered at 1531, 1551, 1571 and $1591 \mathrm{~nm}$ respectively, for 16-Channel WDM system using DML-T/SMF (a) and DMLT/DSF (b).

In both cases, each channel presents a different optimum $\mathrm{P}_{c h}$. Thus, by means of the $\mathrm{P}_{\mathrm{ch}}$ management of each channel it is possible to reach the $\mathrm{Q}_{\max }$ and enhances WDM system performance can be achieving.

As an example; if a 16-Channel WDM system is designed using DML-T and SMF with channel powers equal to the optimum channel power Pch. all 16 channels will have a $Q$ higher than 8 , corresponding to a BER lower than 10-15. In contrast, if a system design with equal channel power is used some of channels (higher dispersive channels) will fail after propagation through SMF fiber.

In Case $\mathrm{D}$, in order to guarantee a $Q$-Factor=15, the output power laser of the channels centered at 1531, 1551, 1571 and $1591 \mathrm{~nm}$ should be 3.2, 3.5 $3.84 \mathrm{~mW}$ respectively. Such difference is due to the different fiber dispersion coefficients that would be associated to every one of them, as shown in Table 2. Then, the compensation of the dispersion would happen for different chirp values and therefore for different output power values.

From another point of view, if the system were designed with the same value of output power in every laser, there is the risk for the channel with the bigger dispersion value not to exceed the minimum criteria that assure an error-free transmission. 


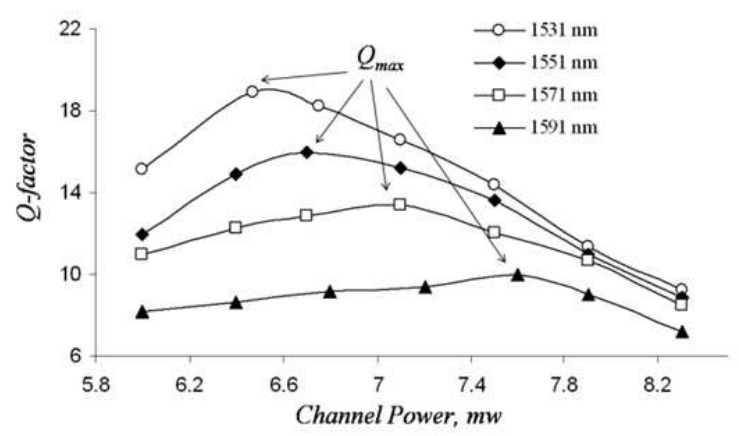

(a) Case C (DML-T/SMF)

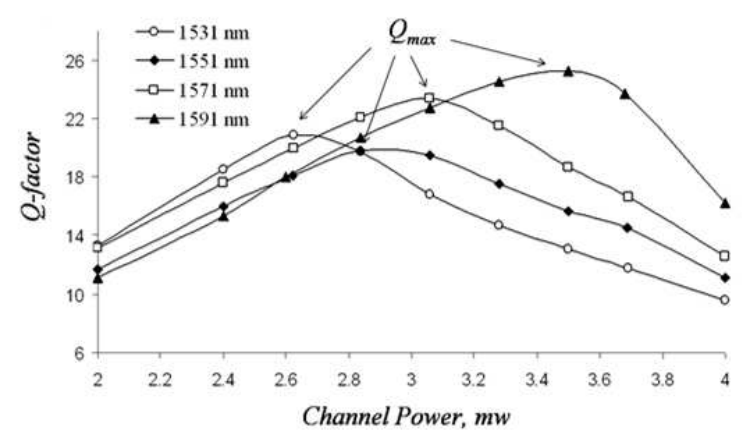

(b) Case D (DML-T/NZ-DSF)

Fig. 18. Q-Factor vs channel power for channels centered at 1531, 1551, 1571 and $1591 \mathrm{~nm}$ respectively, for 16-Channel WDM system using DML-T/SMF (a) and DML-T/NZ-DSF (b).

\begin{tabular}{|l|c|}
\hline Channel & Dispersion \\
\hline $1531 \mathrm{~nm}$ & $15,21 \mathrm{ps} / \mathrm{nm} \cdot \mathrm{km}$ \\
\hline $1551 \mathrm{~nm}$ & $16,34 \mathrm{ps} / \mathrm{nm} \cdot \mathrm{km}$ \\
\hline $1571 \mathrm{~nm}$ & $17,47 \mathrm{ps} / \mathrm{nm} \cdot \mathrm{km}$ \\
\hline $1591 \mathrm{~nm}$ & $18,56 \mathrm{ps} / \mathrm{nm} \cdot \mathrm{km}$ \\
\hline
\end{tabular}

Table 2. Chromatic dispersion of differents channels (SMF fiber)

Since the optimum power channel depends on the global dispersion of the system, a study including the variation of the accumulated dispersion of the global system will be done. The optimum channel powers $\left(\mathrm{P}_{\mathrm{ch}}\right.$ to reach $\left.\mathrm{Q}_{\max }\right)$ are plotted as a function of dispersion in Fig. 19 (open circles in the case of transmission through positive dispersion fiber and solid circles for negative dispersion fiber). In Fig. 19, the results for channel centered at $1551 \mathrm{~nm}$ after transmission over $100 \mathrm{Km}$ of SMF and NZ-DSF fibers as well as a potential CWDM channel centered at $1391 \mathrm{~nm}$ are shown. Attenuation dependence with wavelength was taken account in the calculation of optimum $\mathrm{P}_{\mathrm{ch}}$ and, in all cases, $\mathrm{Q}_{\max }$ higher than 7 (BER lower than 10-12) was obtained. 


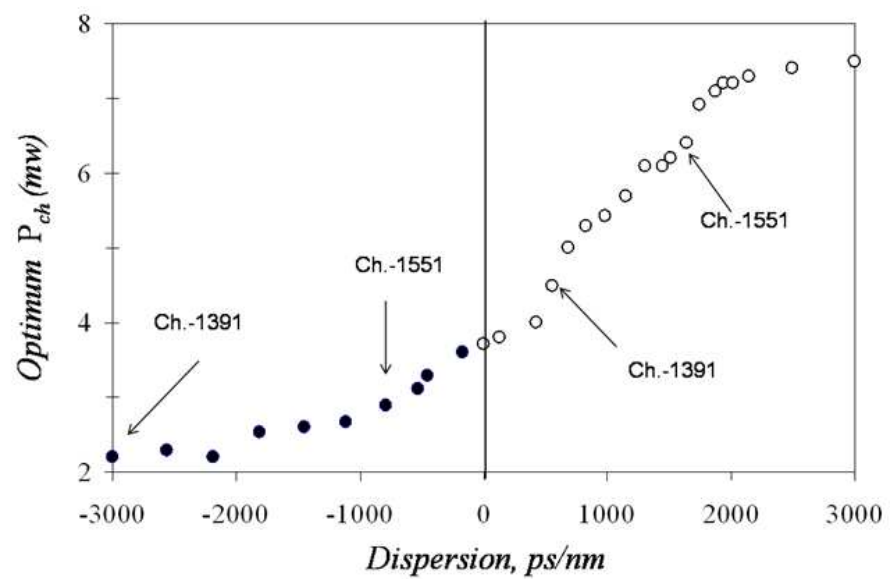

Fig. 19. Comparison of Optimum Channel Powers versus accumulated dispersion for a positive dispersion fiber (open circles) and negative dispersion fiber (solid circles).

In both cases, each channel presents a different optimum $\mathrm{P}_{\text {ch. }}$ Then, by the $\mathrm{P}_{\text {ch }}$. control of each channel it is possible to reach the $Q_{\max }$ and an enhancement of the WDM system performance can be achieved. This optimum $P_{c h}$ is the conclusion of the following considerations: for low power levels, below the optimum power, the Q-Factor increases with Pch because a larger amount of power reaches the detector and the performance enhancement will be dependent upon the level power, so that the greater the power in the receiver, higher system performance is obtained; while, for $\mathrm{P}_{\mathrm{ch}}$ higher than optimum power, the chirp increases with level power and it causes greater frequency shift and linewidth broadening which results in an error in pulse reconstruction.

A mathematical expression that fits this curve would be very useful, since it would make an estimation of the power value to fix the laser output for each channel. For this reason, using the Matlab simulation tool, this function has been estimated from a polynomial expression of degree 4 (Figure 20)

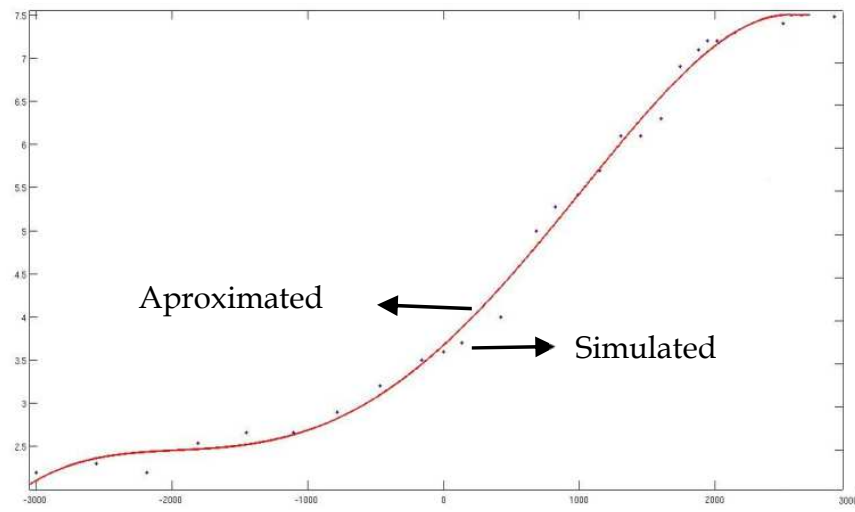

Fig. 20. Estimated and approximated curve 


$$
f(x)=a x^{4}+b x^{3}+c x^{2}+d x+e
$$

$\mathrm{a}=-3.482 \cdot 10-14 ; \mathrm{b}=-6.588 \cdot 10-11 ; \mathrm{c}=4.202 \cdot 10-07 ; \mathrm{d}=0.001435 ; \mathrm{e}=3.673$

where $\mathrm{x}$ is the dispersion accumulated across the link.

Thanks to this equation it is possible to optimize the system behaviour reducing the number of simulations needed for the design stage.

\section{Conclusions}

The performance of fibers relative to positive or negative dispersion characteristics is discussed for the case of directly modulated lasers. The effects of chirp and fiber nonlinearity in a directly modulated $2.5-\mathrm{Gb} / \mathrm{s}$ transmission system have been researched by simulation. We have demonstrated that enhanced system performance, which uses a positive dispersion fiber, can be achieved if positive chromatic dispersion in the optical fiber is equalized by SPM, whereas laser transient chirp can be compensated using a negative dispersion fiber. We can conclude that systems using SMF fiber can have a similar or better performance to those systems that use NZ-DSF fiber if the DML is transient chirp dominated and its output power is properly chosen.

Since the magnitude of SPM can be changed by controlling the optical power in the fiber, the balance between SPM and laser transient chirp can be controlled. Therefore, an optimum compensation condition can be achieved by controlling the optical DML output power. This technique is simple, flexible, and applicable to WDM systems.

In order to analyze the effectiveness of this technique for WDM systems, simulations varying the number of channels from 1 to 16 have been carried out and checking. In every case, $Q$-Factor shows a maximum value depending on the optical power of each channel and accumulated dispersion. This maximum value decreases depending on the number of channels used. Also, we have shown that through the management of the Pch. of each channel it will be possible to enhance the performance of each channel as well as the whole WDM system.

\section{Acknowledgements}

The authors gratefully acknowledge the support of the MICINN (Spain) through project TEC2010-18540 (ROADtoPON).

\section{References}

G.P. Agrawal (2010) Fiber-Optic Communication System. A John Wiley \& Sons Ed.

S. B. Alexander (1997). Optical Communication Receiver Design. SPIE Press/ IEE.

J.C. Cartledge, G.S. Burley. (1989). The Effect of Laser Chirping on Ligthwave System Performance. J. Lightwave Technol., vol. 7, no. 3, pp. 568-573.

L.A. Coldren and S.W. Corzine. (1995) Diode Lasers and Photonic Integrated Circuits. Wiley Series in Microwave and optical Engineering. 
C. del Río and P.R. Horche (2008). Directly modulated laser intrinsic parameters Optimization for WDM Systems, International Conference on Advances in Electronics and Micro-electronics, Sep-Oct 2008.

C. del Río, P. R. Horche, A. Martín Minguez (2010). Effects of Modulation Current Shape on Laser Chirp of $2.5 \mathrm{~Gb} / \mathrm{s}$ Directly Modulated DFB-Laser. Proceedings of The Third International Conference on Advances in Circuits, Electronics and Micro-electronics. CENICS 2010. ISBN.: 979-0-7695-4089-4

C. del Río, P.R. Horche, and A. M. Minguez (2010). Effects of Modulation Current Shape on Laser Chirp of $2.5 \mathrm{~Gb} / \mathrm{s}$ Directly Modulated DFB-Laser. Proc. Conf. on Advances in Circuits and Micro-electronics, pp. 51-55, CENICS 2010

GVD effects in fiber optic communications: dispersion- and power-map cooptimization using genetic algorithm, Optical Engg., volume 47, pp. 075003.

Hamza, M. Y., Tariq, S., Awais, M. M. \& Yang, S. (2008). Mitigation of SPM and

Henry, C. H. (1982), Theory of the linewidth of semiconductor lasers, IEEE J.Quantum Electron.,QE-18(2), 259-264.

K. Hinton and T. Stephens. (August 1993). Specifying Adiabatic Lasers for 2-5gbitls. High Dispersion IM/DD Optical Systems. Electronics Letters. Vol. 29 No. 16.

P. Horche and Carmina del Río (2008). Enhanced Performance of WDM Systems using Directly Modulated Lasers on Positive Dispersion Fibers. Optical Fiber Technology. Volume 14, Issue 2, April 2008, Pages 102-108.

Krelik P. (2006)Characterization of semiconductor laser frequency chirp based on signal distorsion in dispersive optical fiber". Opto-electronics review. vol. 14, no.2, pp. 123-128.

J.A. P. Morgado, and A.V. T. Cartaxo (October 2003). Directly Modulated Laser parameters Optimization for Metropolitan Area Networks Utilizing Negative Dispersion Fibers. IEEE Journal of Selected Topics in Quantum Electronics, Vol. 9, No. 5.

M. Osinski and J. Buss (January 1987). Linewidth broadening factor in semiconductor lasers - An overview, IEEE J. Quantum Electron., vol. 23, no. 1, pp. 9-29,.

E. Peral, W. K. Marshall and A. Yariv (October 1998). Precise Measurement of Semiconductor Laser Chirp Using Effect of Propagation in Dispersive Fiber and Application to Simulation of Transmission Through Fiber Gratings. Journal of Lightwave Technology, Vol. 16, No. 10,.

D. Rodríguez, I. Esquivias, S. Deubert, J. P. Reithmaier, A. Forchel, M. Krakowski, M. Calligaro, and O. Parillaud. (February 2005 ). Gain, Index Variation, and Linewidth-Enhancement Factor in 980-nm Quantum-Well and Quantum-Dot Lasers. IEEE Journal of Quantum Electronics, Vol. 41, No. 2.

N. Suzuki, and T. Ozeki. (1993). Simultaneous Compensation of Laser Chirp, Kerr Effect, and Dispersion in 10-Gb/s Long-Haul Transmission Systems. J. Lightwave Technol, vol. 11, no. 9, pp. 1486-94.

Tomkos et. al. (2001a). Demonstration of negative dispersion fibers for DWDM metropolitan Area networks, IEEE J. of Select. Top. in Quan. Elec. vol. 7, no. 3, pp. 439-60.

I. Tomkos, I. Roudas, R. Hesse, N. Antoniades, A. Boskovic, R. Vodhanel. (2001b). Extraction of laser rate equations parameters for representative simulations of metropolitanarea transmission systems and networks. Optics-Communications. 194(1-3): 109-29

I. Tomkos, R. Hesse, R. Vodhanel, and A. Boskovic. (March 2002). Metro Network Utilizing 10-Gb/s Directly Modulated Lasers and Negative Dispersion Fiber. IEEE Photon. Technol. Lett., VOL. 14, NO. 3. 
L.-S. Yan, C. Yu, Y.Wang, T. Luo, L. Paraschis, Y. Shi, and A. E. Willner.(2005). 40-Gb/s Transmission Over $25 \mathrm{~km}$ of Negative-Dispersion Fiber Using Asymmetric Narrow-Band Filtering of a Commercial Directly Modulated DFB Laser. IEEE Photon. Technol. Lett., vol. 17, no. 6, pp. 1322-1324. 


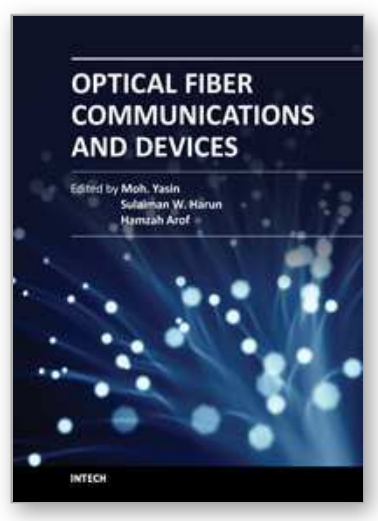

\author{
Optical Fiber Communications and Devices \\ Edited by Dr Moh. Yasin
}

ISBN 978-953-307-954-7

Hard cover, 380 pages

Publisher InTech

Published online 01, February, 2012

Published in print edition February, 2012

This book is a collection of works dealing with the important technologies and mathematical concepts behind today's optical fiber communications and devices. It features 17 selected topics such as architecture and topologies of optical networks, secure optical communication, PONs, LANs, and WANs and thus provides an overall view of current research trends and technology on these topics. The book compiles worldwide contributions from many prominent universities and research centers, bringing together leading academics and scientists in the field of photonics and optical communications. This compendium is an invaluable reference edited by three scientists with a wide knowledge of the field and the community. Researchers and practitioners working in photonics and optical communications will find this book a valuable resource.

\title{
How to reference
}

In order to correctly reference this scholarly work, feel free to copy and paste the following:

Carmina del Río Campos and Paloma R. Horche (2012). Effects of Dispersion Fiber on CWDM Directly Modulated System Performance, Optical Fiber Communications and Devices, Dr Moh. Yasin (Ed.), ISBN: 978953-307-954-7, InTech, Available from: http://www.intechopen.com/books/optical-fiber-communications-anddevices/effects-of-dispersion-fiber-on-cwdm-directly-modulated-system-performance

\section{INTECH}

open science | open minds

\section{InTech Europe}

University Campus STeP Ri

Slavka Krautzeka 83/A

51000 Rijeka, Croatia

Phone: +385 (51) 770447

Fax: +385 (51) 686166

www.intechopen.com

\section{InTech China}

Unit 405, Office Block, Hotel Equatorial Shanghai

No.65, Yan An Road (West), Shanghai, 200040, China

中国上海市延安西路65号上海国际贵都大饭店办公楼 405 单元

Phone: +86-21-62489820

Fax: +86-21-62489821 
(C) 2012 The Author(s). Licensee IntechOpen. This is an open access article distributed under the terms of the Creative Commons Attribution 3.0 License, which permits unrestricted use, distribution, and reproduction in any medium, provided the original work is properly cited. 